\title{
Three tandemly aligned $L E A$ genes from Medicago truncatula confer differential protection to Escherichia coli against abiotic stresses
}

\author{
Y.-M. ZHANG ${ }^{1,3}$, H.-Q. WANG ${ }^{1,2,4 *}$, D.-M. LIU ${ }^{1,2,4}$, and R.-J. LIU ${ }^{1,2,4}$ \\ Northwest Institute of Plateau Biology, Chinese Academy of Sciences, Xining 810001, P.R. Chinal \\ Institute of Three-River-Source National Park, Chinese Academy of Sciences, Xining 810001, P.R. China ${ }^{2}$ \\ University of the Chinese Academy of Sciences, Beijing 100081, P.R. China ${ }^{3}$ \\ Qinghai Provincial Key Laboratory of Crop Molecular Breeding, Xining 810001, P.R. China ${ }^{4}$
}

\begin{abstract}
Late embryogenesis abundant (LEA) proteins are important for abiotic stress tolerance in diverse organisms. Within the LEA protein superfamily, group 4 members are characterized by a conserved N-terminal region and a structurally disordered C-terminal region that varies regarding length and amino acid content. Previous in vitro assays have suggested that the conserved N-terminal region shared by group 4 LEA proteins is critical for forming an amphipathic $\alpha$-helix and protecting enzymatic activities from the adverse effects of desiccation or freezing. However, the cellular roles of the varying C-terminal region remain largely to be characterized. Medicago truncatula contains five subgroup LEA4B proteins encoding loci of which three are tandemly arranged on chromosome 7 due to local gene duplication events. In this study, abiotic stresses and addition of abscisic acid (ABA) induced the transcription of the four $L E A 4 B$ genes. Escherichia coli cells overexpressing the three tandemly aligned $L E A$ genes indicated significantly increased tolerance to salt, osmotic, heat, and freezing stresses. However, the extent of the protective effects on the survival and growth of bacterial cells differed among the LEA proteins, potentially because of variations in the C-terminal region. This possibility was further supported by the observation that the protective effects of the native truncated MtLEA3140, which only contains a conserved N-terminal region, were inferior to the effects of the full-length mutant version. The results suggest that the structurally flexible C-terminal region of group 4 LEA proteins plays roles in protecting cells from damages caused by various abiotic stresses, and provide clues for elucidating the mechanisms underlying the intracellular functions of these proteins.
\end{abstract}

Additional key words: abscisic acid, barrel medic, cold, C-terminal region, Escherichia coli, heat, late embryogenesis abundant protein, salinity.

\section{Introduction}

Late embryogenesis abundant (LEA) proteins were originally found during the late developmental stages of cotton seeds undergoing desiccation (Dure et al. 1981, 1989), but were subsequently observed to accumulate in plant vegetative organs in response to dehydrationinduced stresses, such as drought, salinity, and cold, or to the addition of abscisic acid (ABA) (Battaglia et al. 2008, Hundertmark and Hincha 2008). Further identification of LEA suggested that these proteins are widely distributed across archaeal, eubacterial, and eukaryotic domains, and are not exclusive to plants. Considerable evidence has suggested that LEA proteins are important for tolerance of diverse organisms to abiotic stresses (Battaglia et al. 2008, Hundertmark and Hincha 2008). Moreover, several lines of evidence proposed involvement of LEA protein in biotic stress responses (Salleh et al. 2012, Yang et al. 2012, Liu et al. 2013, Mota et al. 2019).

The LEA proteins have been divided into several groups based on their sequence similarities and conserved motifs, although different groupings and nomenclatures have been

Submitted 21 March 2019, last revision 26 July 2019, accepted 2 September 2019.

Abbreviations: ABA - abscisic acid; cDNA - complemental DNA; CFU - colony forming unit; GRAVY - grand average of hydropathy; IPTG - isopropyl $\beta$-D-1-thiogalactopyranoside; LB medium - Luria-Bertani medium; LEA - late embryogenesis abundant; PCR polymerase chain reaction; PTC - premature termination codon; RT-PCR - reverse transcription PCR; SDS-PAGE - sodium dodecyl sulfate polyacrylamide gel electrophoresis; SNP - single nucleotide polymorphism.

Acknowledgements: This work was supported by the National Natural Science Foundation of China (Grant no. 31770365), the Applied Basic Research Foundation of Qinghai Province (Grant no. 2017-ZJ-784) and the Construction Project for Innovation Platform of Qinghai Province (Grant no. 2017-ZJ-Y14).

*Corresponding author; fax: (86) 971 6143282; e-mail: wanghq@nwipb.cas.cn 
used (Dure et al. 1989, Bray 1993, Dure 1993, Wise 2003, Battaglia et al. 2008). The classification of LEA proteins proposed by Battaglia et al. (2008) categorized LEA proteins into seven groups, of which group 4 comprises members characterized by a conserved $\mathrm{N}$-terminal region with 70 - 80 amino acids tending to form an amphipathic $\alpha$-helix as well as a structurally disordered C-terminal region that varies in length and amino acid content (Battaglia et al. 2008, Olvera-Carrillo et al. 2010, CuevasVelazquez et al. 2016). The group 4 LEA proteins were further divided into subgroup $4 \mathrm{~A}$ and subgroup 4B. In Arabidopsis thalianana, there are three LEA 4 proteins, of which one belongs to subgroup 4A and two are members of subgroup 4B. The phenotyping of Arabidopsis thaliana mutants deficient in the group 4 LEA proteins and LEAoverexpressing transgenic plants indicated that these LEA proteins contribute to plant tolerance to water stress (Olvera-Carrillo et al. 2010). In vitro assays have revealed multiple functions for the group 4 LEA proteins, including alleviating dehydration- or freezing-induced membrane damage and protein inactivation, binding of sugar or metals, and scavenging of radicals (Reyes et al. 2005, 2008, Hundertmark et al. 2011, Liu et al. 2011, Dang et al. 2014). The results of in vitro assays suggest that the conserved $\mathrm{N}$-terminal region of group 4 LEA proteins is critical for the conformational transitions induced by water deficit stress and for the protection of enzyme activities (Reyes et al. 2008, Cuevas-Velazquez et al. 2016). However, the potential roles of the variable $\mathrm{C}$-terminal region in vivo remain largely unknown. Previous identification and our search against Medicago truncatula genome revealed five loci that encode group 4 LEA proteins. Of the five LEA proteins, three showing a highly conserved N-terminal region and a varying $\mathrm{C}$-terminal region are encoded by tandemly aligned loci on chromosome 7 of Medicago truncatula, offering advantages for characterization of roles played by the C-ternimal region of group 4 LEA proteins.

In this study, the three tandemly aligned group 4 LEA protein-encoding genes (MtLEA3140, MtLEA3160, and MtLEA370) in the Medicago truncatula genotype R108 will be isolated and overexpressed in Escherichia coli cells. The data presented herein may be useful for elucidating the effects of the structurally disordered $\mathrm{C}$-terminal region of group 4 LEA proteins on intracellular functions in response to abiotic stresses.

\section{Materials and methods}

Plants, growth conditions, and treatments: Barrel medic (Medicago truncatula Gaernt.) genotypes Jemalong A17 (A17) and R108 were used for transcription profiling, while R108 was also used for the cloning of $L E A$ genes. Seeds were treated with concentrated sulfuric acid for $10 \mathrm{~min}$ and then rinsed thoroughly with water. The scarified seeds were placed on wet filter paper in Petri dishes and incubated at a temperature of $4{ }^{\circ} \mathrm{C}$ under darkness for $3 \mathrm{~d}$. The seeds were then incubated in a growth chamber at $21{ }^{\circ} \mathrm{C}$, a 16 -h photoperiod, an irradiance of
$120 \mu \mathrm{mol} \mathrm{m} \mathrm{m}^{-2} \mathrm{~s}^{-1}$, and a relative humidity of $70-75 \%$ to induce germination. After 1 week, the seedlings were transplanted to Vermiculite and watered once a week with a solution containing $2 \%$ NutriSmart 302 fertilizer (CK Life Sciences, Hong Kong, China).

Seedlings grown in Vermiculite for 2 weeks were subjected to abiotic stresses and ABA treatments. For cold treatment, the seedlings were subjected to $4{ }^{\circ} \mathrm{C}$. For dehydration, the seedlings were removed from the Vermiculite and dehydrated in air at $21{ }^{\circ} \mathrm{C}$. Salinity and ABA treatments were carried out by transferring the seedlings to $200 \mathrm{mM} \mathrm{NaCl}$ solution or spraying with $50 \mu \mathrm{M}$ ABA solution, respectively. Samples were collected at appropriate time points and immediately frozen in liquid nitrogen for extraction of total RNAs.

Reverse transcription PCR and transcriptional

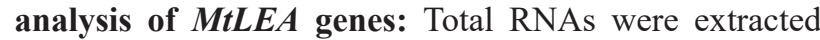
with TRIzol reagent (Gibco, Grand Island, NY, USA), followed by treatment with RNase-free DNase I (Takara, Dalian, China). First-strand cDNAs were synthesized using $M-M L V$ reverse transcriptase (Sangon, Shanghai, China). The MtLEA3140,3160, and 3170 coding sequences were amplified by PCR using first-strand cDNAs from seedlings of R108 that underwent a 24-h cold treatment and the primer sets listed in Table 1 Suppl. with highfidelity Pyrobest DNA polymerase (Takara). Amplicons were cloned into the SmaI site of the pBluescript II $S K(+)$ vector (Stratagene, La Jolla, CA, USA) for sequencing confirmation. At least three independent clones for each of the genes were randomly chosen for sequencing. Sequence analysis of the MtLEAs was carried out with DNAMAN software (Lynnon Biosoft, Quebec, Canada).

To examine the effects of abiotic stresses and ABA on the transcription of MtLEA genes, first-strand cDNAs from genotype R108 and A17 were synthesized with the PrimeScript RT reagent kit (Takara). The subsequent PCR amplification was completed using the primer sets as described above. An actin gene (Medtr7g026230; Table 1 Suppl., Zhang et al. 2011) was included as an internal control. The PCR products were detected by $0.7 \%(\mathrm{~m} / \mathrm{v})$ agarose gel electrophoresis.

Site-directed mutagenesis of MtLEA3140 and heterologous expression of MtLEAs in Escherichia coli: MtLEA3140 from genotype R108 cloned in pBluescript II $S K(+)$ was used as a template for site-directed mutagenesis with the primer set listed in Table 1 Suppl. and the QuikChange Site-Directed mutagenesis kit (Stratagene). The mutagenesis replaced the codon responsible for the PTC (TAG) with CAG based on the corresponding fulllength gene sequence in genotype A17. The resulting mutant sequence was named MtLEA3140M.

For the heterologous expression of MtLEA genes in Escherichia coli cells, the open reading frames of the three MtLEA genes and the MtLEA3140M (mutant version of MtLEA3140) were ligated into the BamHI/SacI sites of pET-30a (Novagen, town, Germany). The resulting plasmids were introduced into E. coli BL21 (DE3) cells (Novagen). Recombinant protein production was induced 
by addition of isopropyl $\beta$-D-1-thiogalactopyranoside (IPTG; final concentration of $0.5 \mathrm{mM}$ ) into bacterial cultures at the exponential growth stage. The cultures were subsequently incubated at $37^{\circ} \mathrm{C}$ for $3 \mathrm{~h}$, after which $1 \mathrm{~cm}^{3}$ of culture aliquots were centrifuged at $12000 \mathrm{~g}$ for $1 \mathrm{~min}$. The pellets were resuspended in $0.1 \mathrm{~cm}^{3}$ of $1 \times$ sodium dodecyl sulfate polyacrylamide gel electrophoresis (SDSPAGE) sample buffer followed by incubation in boiling water for $10 \mathrm{~min}$. The boiled samples were centrifuged at $12000 \mathrm{~g}$ for $10 \mathrm{~min}$ at room temperature, and the supernatants were analyzed by $12 \%(\mathrm{~m} / \mathrm{v})$ SDS-PAGE. To examine the heat tolerance of MtLEA proteins, the pellets of IPTG-induced cultures were resuspended in $0.08 \mathrm{~cm}^{3}$ of double-distilled water instead of SDS-PAGE sample buffer and then incubated in boiling water for $30 \mathrm{~min}$. After centrifugation at $12000 \mathrm{~g}$ for $10 \mathrm{~min}$, the supernatants of the boiling water extracted samples were collected for preparation of SDS-PAGE samples. The identities of the recombinant MtLEA proteins were dected by Western blotting using an anti-His $\times 6$ antibody (Boster, Wuhan, China) and an enhanced chemiluminescence kit (Yeasen, Shanghai, China).

Analysis of the abiotic stress tolerance of Escherichia coli cells overexpressing MtLEAs: To determine the effects of MtLEA proteins on the tolerance of E. coli cells to abiotic stresses, IPTG-induced cultures were diluted with liquid Luria-Bertani (LB) medium to an absorbance

A

MtLEA3140
MtLEA3160
MtLEA3170
AtLEA4-5

Medtr1g053970

Medtr4g098480

AtLEA4-1

AtLEA4-2

MtLEA3140

MtLEA3160

MtLEA3170

AtLEA4-5

Medtr1g053970

Medtr4g098480

AtLEA4-1

AtLEA4-2

MtLEA3140

MtLEA3160

MtLEA3170

AtLEA4-5

Medtr1g053970

Medtr4g098480

AtLEA4-1

AtLEA4-2

$B$

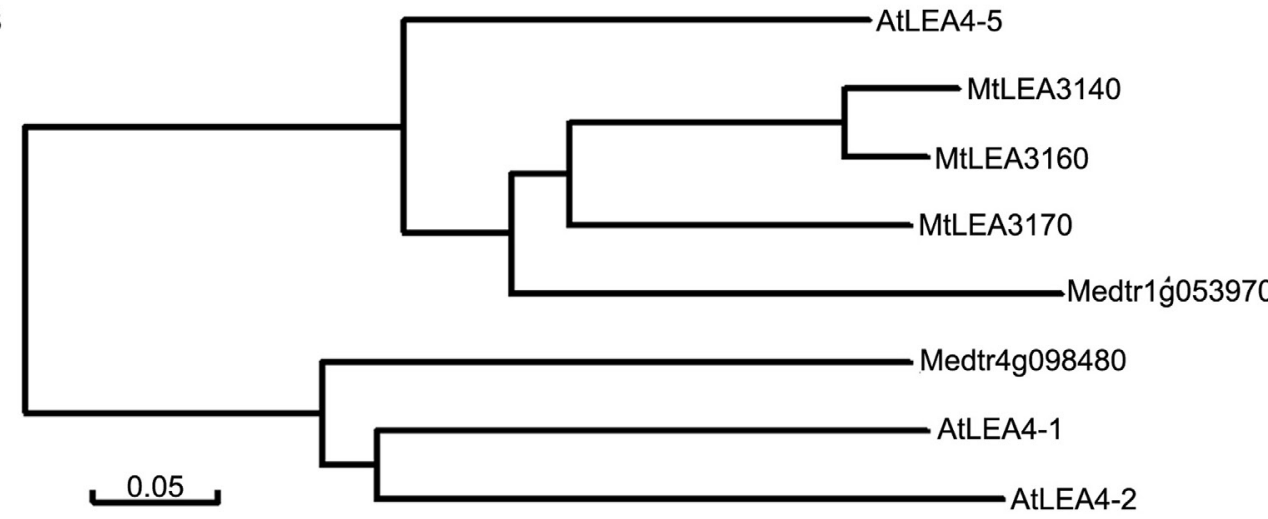

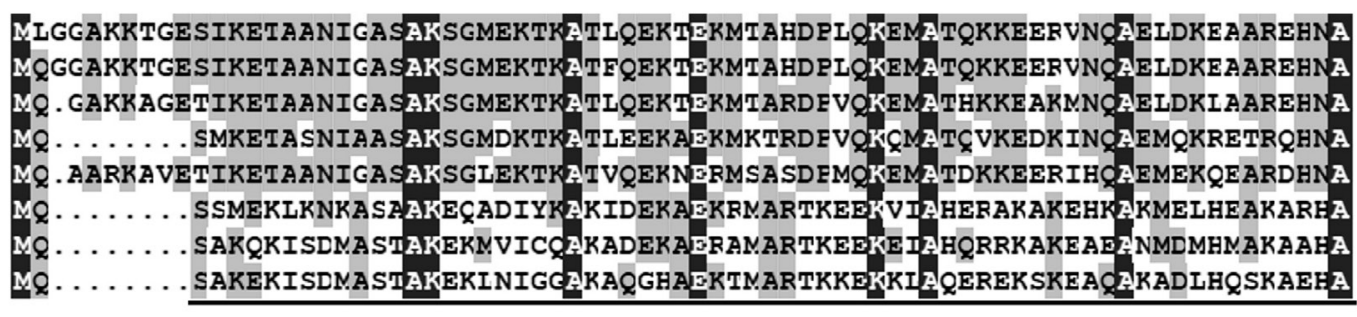

75

75

74 67 74 67 67 67

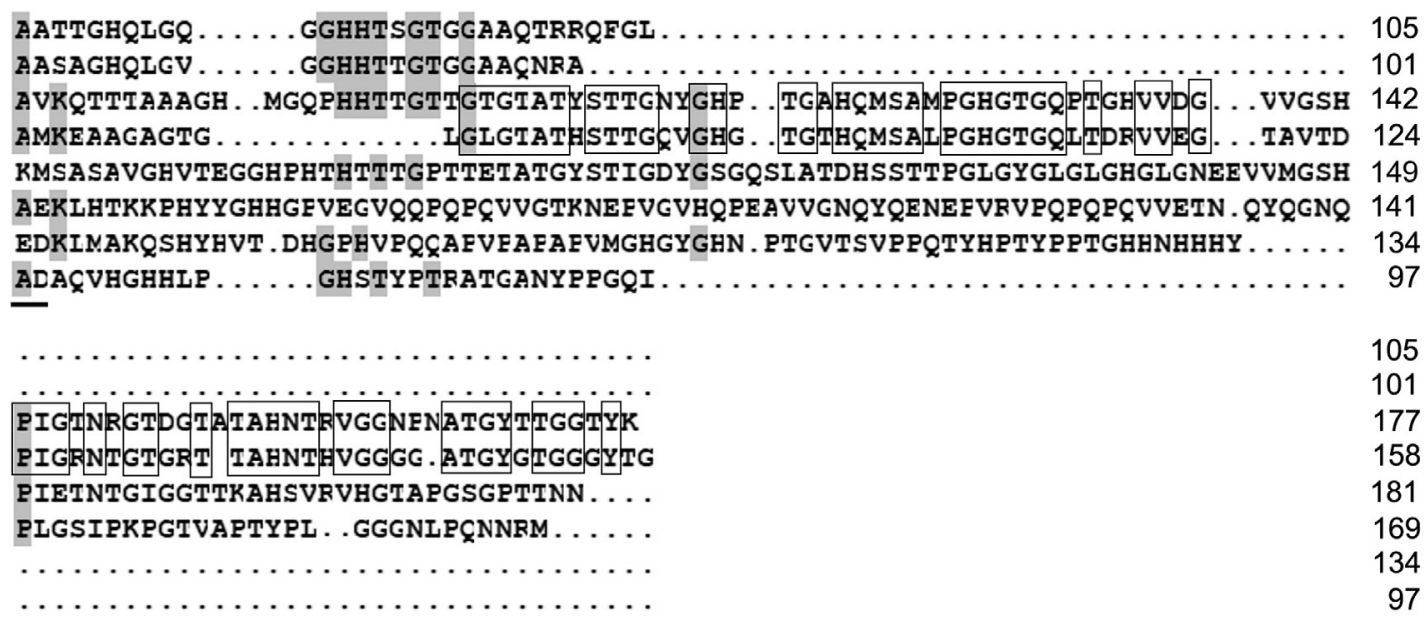

PIGINRGTDGT TAHNTRVGGNFN ATGYYTTGGTYK PIGRNIGTGRT TAHNTEVGGGG. ATGYGTGGGYTG 169 134 97

Fig. 1. Sequence analysis of group 4 late embryogenesis abundant (LEA) proteins from Medicago truncatula and Arabidopsis thaliana. $A$ - Alignment of amino acid sequences of LEA proteins. $M$. truncatula LEA protein sequences were deduced from the genotype A17 reference genome. The conserved N-terminal regions of group 4 LEA proteins are underlined. The conserved amino acid residues in the C-terminal regions of MtLEA3170 and AtLEA4-5 are framed. $B$ - Phylogenetic tree presenting the two subgroups of group 4 LEA proteins. 


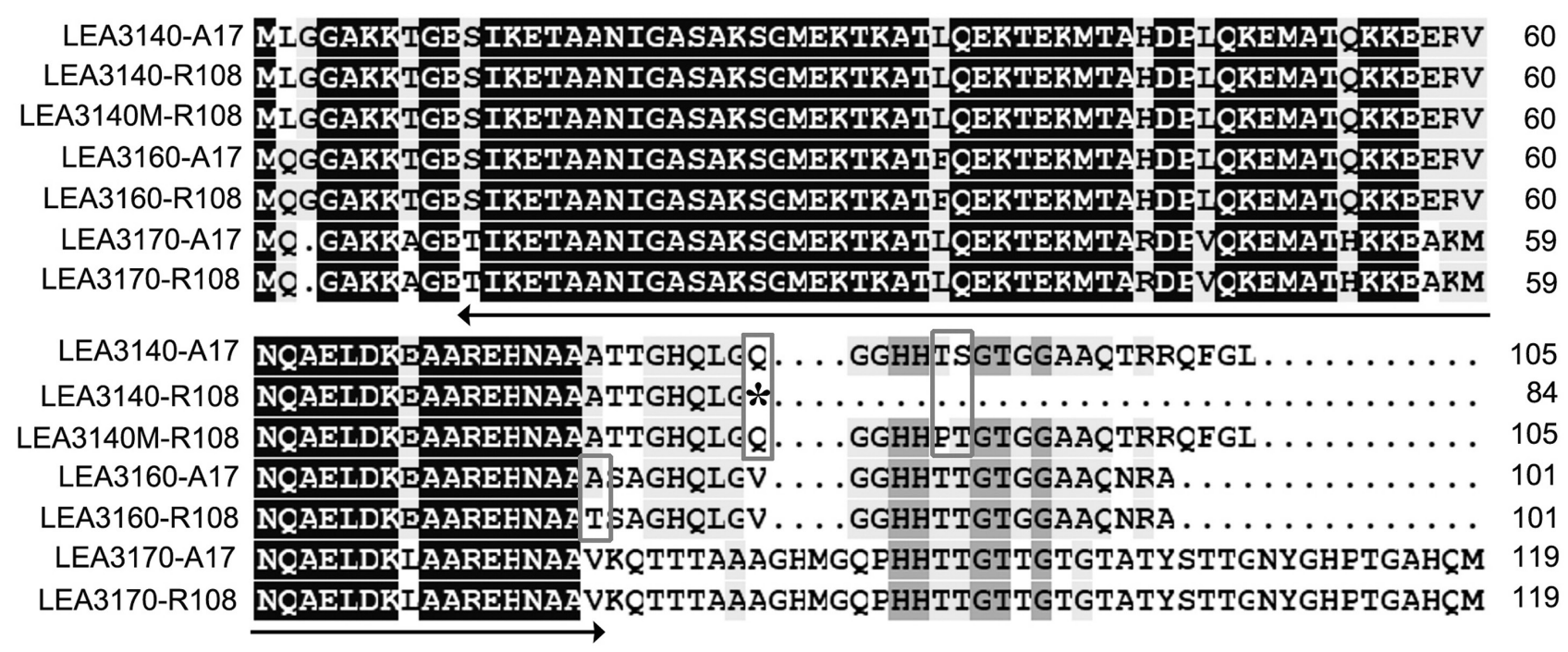

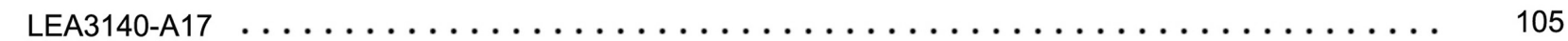

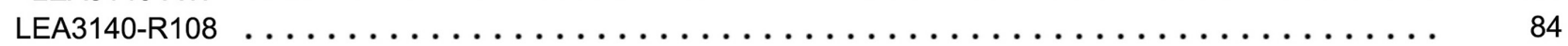

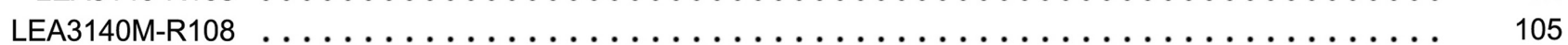

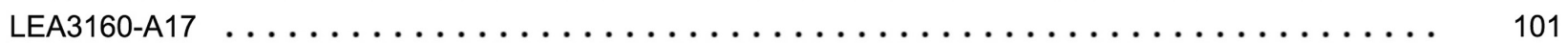

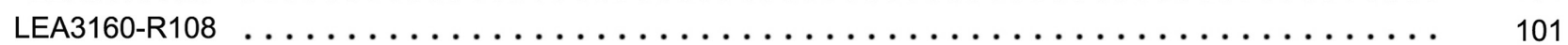

LEA3170-A17 SẠMFGHGTGQPTGHVVDGVVGSHPIGTNRGTDGTATAHNTRVGGNENATGYTTGGTYK 177

LEA3170-R108 SP̣MPGHGTGQPTGHVVDEVVGSHPIGTNRGTDGTATAHNTFVGGNFNATGYTTGGTYK 177

Fig. 2. Sequences of three MtLEA4B proteins encoded by tandemly aligned loci in Medicago truncatula genotypes A17 and R108. The conserved $\mathrm{N}$-terminal regions are indicated with a double-arrowed line. The premature termination codon introduced by a single nucleotide polymorphism is indicated by an asterisk, while amino acid substitutions are framed. MtLEA3140M-R108, which is the sitedirected mutant version of MtLEA3140 from R108, is included.

$\left(\mathrm{A}_{600}\right)$ of 0.9 . The cultures were then serially diluted 10-fold in liquid LB medium. For salinity and dehydration treatments, $10 \mathrm{~mm}^{3}$ of serially diluted cultures were dotted to kanamycin $\left(50 \mathrm{mg} \mathrm{dm}^{-3}\right)$ and IPTG $(0.5 \mathrm{mM})$ contained agar-solidified LB plates with addition of $500 \mathrm{mM} \mathrm{NaCl}$ or $\mathrm{KCl}$ (salinity), or $500 \mathrm{mM}$ mannitol (osmotic stress). After incubation at $37^{\circ} \mathrm{C}$ for $48 \mathrm{~h}$, the colony growth on the plate was photographed with a digital camera. Heat and freezing treatments were performed by incubating the serially diluted IPTG-induced cultures at $55^{\circ} \mathrm{C}$ for $30 \mathrm{~min}$ and freezing at $-20^{\circ} \mathrm{C}$ for $12 \mathrm{~h}$, respectively, followed by dotting $10-\mathrm{mm} 3$ aliquots of the heat or freezing treated cultures to kanamycin contained LB plates for incubation at $37{ }^{\circ} \mathrm{C}$ overnight. To quantify the colony growth, the serially diluted cultures were spread over LB plates, and the resulting colonies were counted. Colony survival or growth was determined by calculating the relative colonyforming units (CFUs) [\%] = (colony number in stressed cultures / colony numeber in unstressed cultures) $\times 100$. Data for each of abiotic stress treatments were analyzed with a one-way $A N O V A$ followed by Duncan's multiple test at $P<0.01$.

\section{Results}

A previous genome-wide survey revealed four M. truncatula group 4 LEA members that were divided into two subgroups (Battaglia and Covarrubias 2013). One member (locus Medtr4g098480) belonging to subgroup 4A encodes a relatively varying amino acid sequence (Fig. 1). The remaining four members of subgroup $4 \mathrm{~B}$, encode amino acid sequences with a highly conserved N-terminal region and a $\mathrm{C}$-terminal region that varies regarding amino acid sequence or length (Fig. 1). Here, further search against genome of Medicago truncatula found an additional subgroup 4B member encoded by the locus of Medtrlg053970 (Fig. 1). Of the four subgroup 4B member loci, there are three occurring as tandemly aligned loci on chromosome 7, Medtr7g093140, Medtr7g093160, and Medtr $7 g 093170$ that are denoted herein as MtLEA3140, $M t L E A 3160$, and $M t L E A 3170$, respectively.

Three tandemly arranged LEA genes from genotype R108 were cloned and sequenced. In comparisons with the corresponding gene sequences in the genotype A17 reference genome, three, two, and one SNPs were detected in MtLEA3140, MtLEA3160, and MtLEA3170, respectively (Fig. 1 Suppl.). In MtLEA3140, one of the SNPs (C-to-T transition) generates a premature termination codon (PTC), while the remaining two transversions (i.e., A-to-C and T-to-A) result in amino acid substitutions (i.e., Thr to Pro and Ser to Thr, respectively) (Fig. 2 and Fig. 1 Suppl.). Additionally, one of the two G-to-A transitions in $M t L E A 3160$ is responsible for an amino acid substitution (Ala to Thr), while the other transitions (G-to-A in MtLEA3160 as well as C-to-T in MtLEA3170) do not 


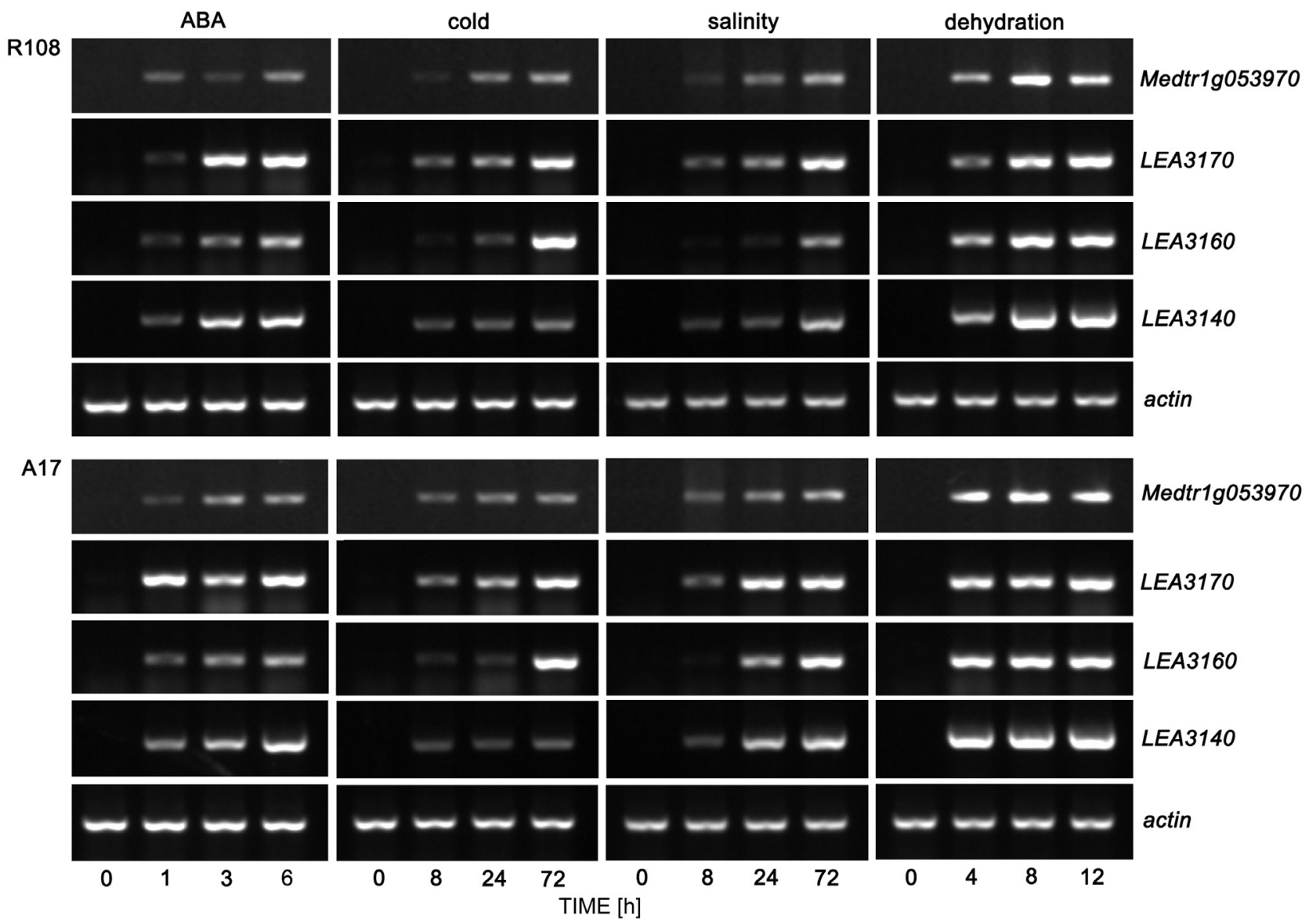

Fig. 3. The reverse transcription PCR detection of transcription of MtLEA4B genes in Medicago truncatula genotype A17 and R108 in response to abiotic stresses and abscisic acid.

result in amino acid substitutions because of their in-frame synonymous substitutions (Fig. 2 and Fig. 1 Suppl.).

The deduced amino acid sequences indicated that the estimated molecular masses for MtLEA3160 and MtLEA3170 were 10.6 and $18.1 \mathrm{kDa}$, respectively, whereas the grand average of hydropathy (GRAVY) indices were -1.067 and -0.864 , respectively. Because of the presence of a PTC, MtLEA3140 from genotype R108 encodes a truncated protein consisting of 84 amino acids, with an estimated molecular mass of $9.0 \mathrm{kDa}$. This is in contrast to the corresponding full-length mutant version, which comprises 105 amino acids, with an estimated molecular mass of $10.0 \mathrm{kDa}$. The GRAVY indices of the native truncated MtLEA3140 from R108 and its full length mutant version MtLEA3140M (Fig. 2) are both -1.036.

To determine the transcriptional responses of MtLEA3140, MtLEA3160, MtLEA3170 and the locus of Medtrlg053970 to abiotic stresses, M. truncatula seedlings were treated with salinity, desiccation, heat, cold, and ABA. The resulting transcription patterns of the four $L E A 4 B$ genes were analyzed by RT-PCR. The transcription of these four genes from both genotypes A17 and R108 was apparently induced by the abiotic stresses and ABA in a time-dependent manner (Fig. 3), implying that the genes are involved in responses to various abiotic stresses. Moreover, no diurnal transcription oscillations of the $M t L E A$ genes were detected under normal condition (Fig. 2 Suppl.), eliminating the effect of photoperiod or circadian rhythm on their expression patterns. Most LEA proteins remained soluble after being heated because they are intrinsically unstructured (Receveur-Bréchot et al. 2006, Rivera-Najera et al. 2014). To assess the stability of heat-treated LEA proteins, the three MtLEA proteins encoded by the tandemly aligned loci on chromosome 7 from genotype R108 and a full-length mutant version of LEA3140, LEA3140M (Fig. 2), were overexpressed in $E$. coli cells. The IPTG treatment of bacterial cultures with cells carrying the $L E A$-expression vectors resulted in an apparently extra band in the SDS-PAGE gels while a weak band corresponding to each of the recombinant proteins was also detected in the cultures without addition of IPTG due to leaking expression of the MtLEAs (Fig. 4A). The apparent molecular masses of the recombinant LEA proteins were higher than the estimated molecular masses (Fig. 4) because of the high hydrophilicity or disordered structure characterized by most hydrophilins, including LEA proteins (Receveur-Bréchot et al. 2006, Rivera-Najera et al. 2014). However, LEA3160 exhibited slightly greater retarded migration during the SDS-PAGE analysis than LEA3140M (Fig. 4), even though its estimated molecular mass is lower than that of LEA3140M. Sequencing of $L E A 3160$ and LEA3140M in pET-30a plasmid eliminated 


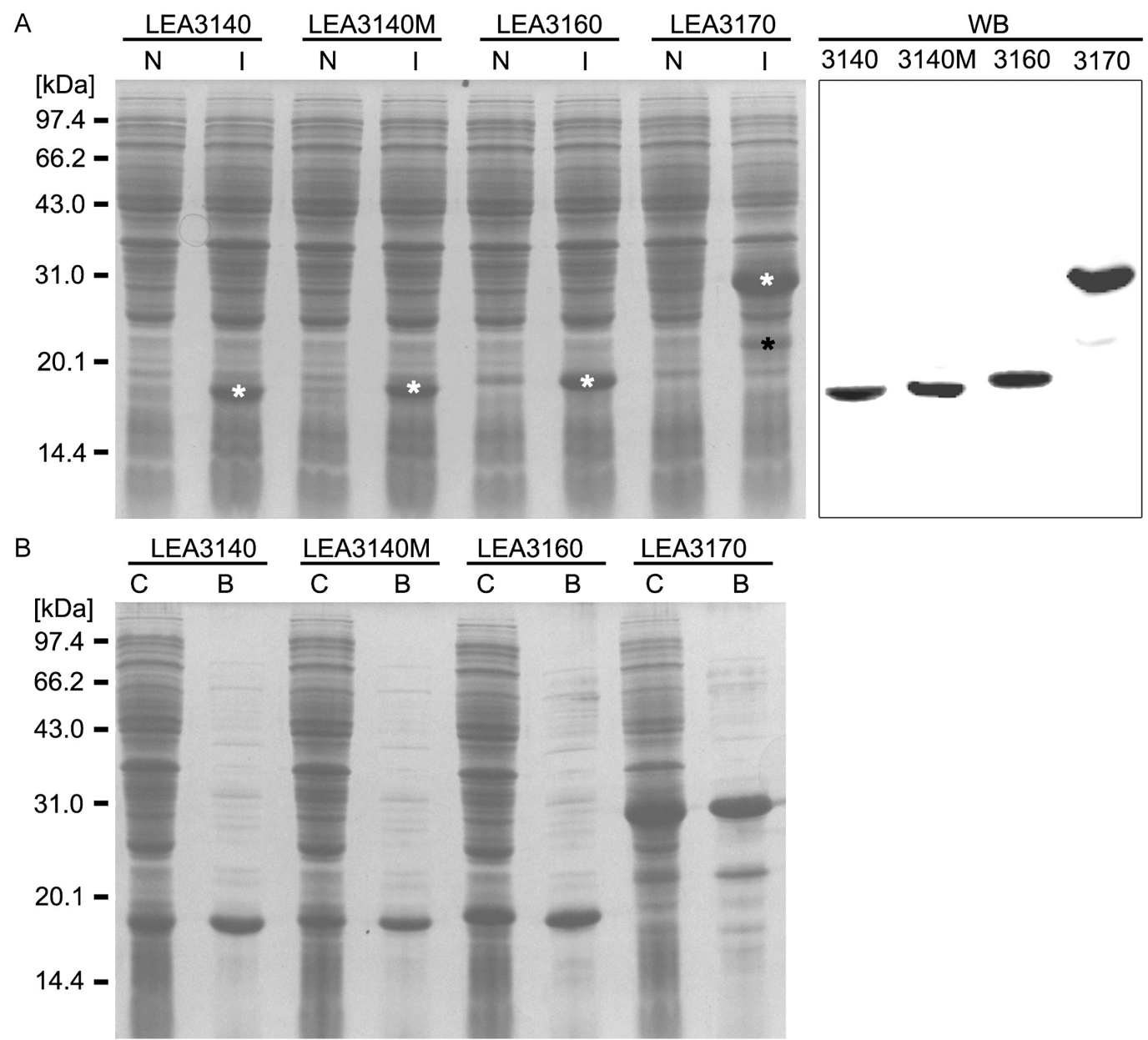

Fig. 4. Heterologous expression of MtLEAs in Escherichia coli and the heat tolerance of the recombinant proteins. $A$ - Total proteins from $E$. coli cultures without $(\mathrm{N})$ and with the addition of isopropyl $\beta$-D-1-thiogalactopyranoside (IPTG) (I). The bands of the recombinant MtLEAs were indicated by white or black asteroids, and further detected with Western blotting (WB) by using of an anti-His $\times 6$ antibody. $B$ - SDS-PAGE sample buffer (C) and boiling water (B) extracted crude proteins from IPTG induced $E$. coli cultures.

the possible experimental mistakes resulting in the unexpected electrophoretic migration. Western-blotting detection of the His-tag fusing at the N-terminal ends of the recombinant LEA proteins further confirmed the identities of the recombinant proteins (Fig. $4 A$ ). Additionally, a weak band with an apparent molecular mass of $21.3 \mathrm{kD}$ was also detected in IPTG-induced LEA3170 sample by Western blotting (Fig. 4A), implying presence of other structurally differed forms of the LEA protein. As expected, incubation of the $E$. coli cultures in boiling water for $30 \mathrm{~min}$ did not result in apparent decrease in the abundance of soluble recombinant proteins, but did cause the abundance of E. coli endogenous proteins to decrease (Fig. 4B).

Bacterial and yeast cells have been widely used to determine the effects of LEA proteins on abiotic stress tolerance (e.g., Hu et al. 2016, Saucedo et al. 2017). In this study, we investigated the survival and growth of E. coli cells overexpressing MtLEA proteins under abiotic stresses. The dot assay and CFU analysis indicated that all the tested MtLEA proteins significantly enhanced the tolerance of $E$. coli cells to salt, osmotic, heat, and freezing stresses (Fig. 5). However, the extent of the tolerance differed among the MtLEA proteins (Fig. 5B), with LEA3160 providing the greatest protection against abiotic stresses (Fig. 5B). The survival and growth rates were higher for the $E$. coli cells producing the mutated full-length LEA3140M than that of its corresponding native truncated LEA 3140 of genotype R108 under abiotic stresses (Fig. 5B). Thus, the 21 amino acids at the C-terminal of LEA3140M (Fig. 2) are important for the protection of E. coli against abiotic stresses. Moreover, cells producing LEA3170, which has the longest C-terminal region of the tested LEA proteins (Fig. 2), exhibited similar tolerance to abiotic stresses as those of cells producing LEA3140, with the exception of an unsignificantly higher relative CFUs under $\mathrm{NaCl}$ and freezing stresses (Fig. 5B).

\section{Discussion}

The group 4 LEA proteins have been phylogenetically divided into subgroups 4A and 4B (Battaglia et al. 2008, Olvera-Carrillo et al. 2010). In A. thaliana, the varying lengths of the $C$-terminal region and the partially 

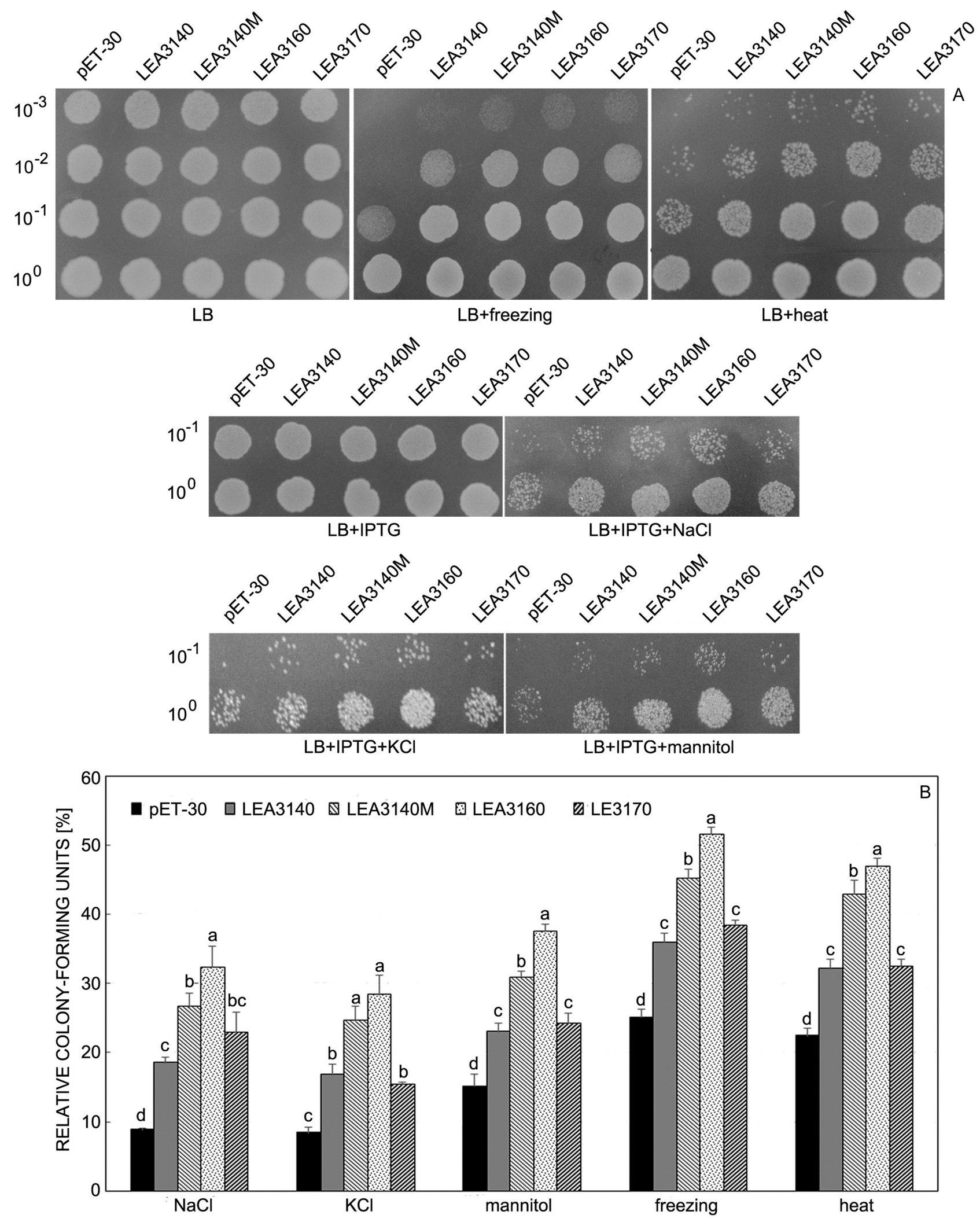

Fig. 5. Abiotic stress tolerance of Escherichia coli cells overexpressing MtLEAs. A - Dot assays. B - Colony-forming unit investigations. Triplicates were averaged and bars indicate standard errors. Different upper case letters indicate statistically significant differences of protective effects among MtLEAs under each of the abiotic stress treatments according to the Duncan's multiple test $(n=3, P<0.01)$. 
overlapping transcription patterns of the members of subgroups $4 \mathrm{~A}$ and $4 \mathrm{~B}$ in response to abiotic stresses suggest their function diversification (Olvera-Carrillo et al. 2010). The $M$. truncatula genome includes one subgroup $4 \mathrm{~A}$ member as well as four subgroup $4 \mathrm{~B}$ members. Of the subgroup 4B, three members are located at tandemly aligned loci on chromosome 7, as described previously (Battaglia et al. 2008) and in this study (Fig. 1). The M. truncatula subgroup 4A member, encoded by the Medtr4g098480 locus, differs somewhat from the A. thaliana LEA 4A proteins (Fig. 1), implying the likelihood of speciesor genus-specific diversification during evolution that needs to be confirmed in future studies. However, the A. thaliana and $M$. truncatula subgroup 4B proteins possess a highly conserved $\mathrm{N}$-terminal region, which is considered to be a signature of group 4 LEA proteins (Fig. 1A). MtLEA3170 and AtLEA4-5 share a relatively long and conserved $\mathrm{C}$-terminal region that differs from the very short C-terminal region of MtLEA3140 and MtLEA3160 (Fig. 1A). Therefore, MtLEA3170 represents an ancient subgroup 4B member of $M$. truncatula, from which MtLEA3140, MtLEA3160, and Medtr1g053970 arose through gene duplication events. The diverse abiotic stresses and ABA induced expression patterns of the four M. truncatula subgroup 4B LEA genes (Fig. 3) suggest their roles in response to various abiotic stresses. However, the transcript of the $M$. truncatula subgroup 4A member encoded by the Medtr4g098480 locus accumulated in response to high salinity and dehydration, while cold stress and ABA did not appear to affect its transcription (Fig. 3 Suppl.). These results further support the previously suggested functional divergence between the two LEA4 subgroups in plants (Battaglia et al. 2008, Olvera-Carrillo et al. 2010).

The heterologous expression of $L E A$ genes in bacterial or yeast cells is an alternative to the time-consuming and labour-intensive generation of transgenic plants for evaluating the effects of LEA proteins on abiotic stress tolerance. Heterologous expression systems may be more useful than the relatively simple in vitro assays for clarifying the cellular functions of LEA proteins. In vitro assays have confirmed that the conserved N-terminal region of Group 4 LEA proteins is necessary and sufficient for the formation of an amphipathic $\alpha$-helix and protection of enzymatic activities under water deficit or freezing (Cuevas-Velazquez et al. 2016, Reyes et al. 2008). However, the evolutionary meaning and in vivo functions of the varying $\mathrm{C}$-terminal region remain relatively uncharacterized. Researchers have speculated that the disordered $\mathrm{C}$-terminal region or terminus may expose some amino acids, such as histidine, which bind to metal ions (Cuevas-Velazquez et al. 2016, 2017). A recent biophysical characterization of metal ion binding properties suggested that the $\mathrm{C}$-terminal region of AtLEA4-5 could function as an antioxidant and a metal ion scavenger under abiotic stresses (French-Pacheco et al. 2018). In this study, we compared protective effects of the three subgroup 4B LEA proteins from M. truncatula on survival and growth of $E$. coli cells under salt, osmotic, heat, and freezing stresses. Despite a previous report describing the bacterial growth inhibition associated with some $A$. thaliana LEA proteins, including LEA4-5 (LEA-D113) (Campos et al. 2006), the overexpression of MtLEA proteins did not obviously affect bacterial cell growth under normal conditions in this study (Fig. 5A). This discrepancy is likely because of differences in the $E$. coli genotypes used in the two studies, although subtle variations in the proteins, such as the extra 7-8 amino acid residues in the C-terminal region of MtLEAs compared to AtLEA4-5, may contribute to the differences in bacterial growth. Bacterial cells overexpressing $M t L E A$ proteins were more viable and survived better than the control cells harbouring an empty vector under abiotic stress treatments (i.e., hyper-osmolarity, high salinity, freezing, and heat) (Fig. 5). Nevertheless, bacterial cells producing MtLEA proteins with different $\mathrm{C}$-terminal regions exhibited varying degrees of tolerance against abiotic stresses (Fig. 5), which was in contrast to the results of previous in vitro assays (Reyes et al. 2008, CuevasVelazquez et al. 2016). Although MtLEA3170 possesses the longest $\mathrm{C}$-terminal region (Figs. 1 and 2) among the three LEA proteins tested here, its protective effect was similar to that of the native truncated MtLEA3140 from genotype R108, which contains the shortest C-terminal region besides with the conserved $\mathrm{N}$-terminal region. However, the highest abiotic stress tolerance observed in this study was conferred by MtLEA3160, which has a very short C-terminal region (Fig. 5). These results suggest the varying $\mathrm{C}$-terminal region is important in protecting organisms against abiotic stresses whereas the possible effects of the variation of the several amino acid residues needs to be confirmed. This suggestion was further supported by the superior protective effects of the full-length mutant MtLEA3140M over the truncated native MtLEA3140 (Fig. 5). Moreover, the results obtained in this study also imply more complicated mechanisms than what have been proposed previously (Cuevas-Velazquez et al. 2016, 2017) in case of the cellular roles played by the C-terminal regions of group LEA4 proteins. Additionally, it is worthy to be noted here that MtLEA3160 exhibited slightly slower electrophoretic migration than LEA3140M (Fig. 4), even though its estimated molecular mass was lower than that of LEA3140M. This result might be due to LEA3160 having a higher hydropathicity or a more flexible structure than LEA3140M. Whether the speculated reasons for the unexpected electrophoretic migration of LEA3160 are associated with the fact that LEA3160 provided the greatest protection against abiotic stresses here (Fig. 5) should be elucidated in future studies.

\section{References}

Battaglia, M., Covarrubias, A.A.: Late embryogenesis abundant (LEA) proteins in legumes. - Front. Plant Sci. 4: 190, 2013.

Battaglia, M., Olvera-Carrillo, Y., Garciarrubio, A., Campos, F., Covarrubias, A.A.: The enigmatic LEA proteins and other hydrophilins. - Plant Physiol. 148: 6-24, 2008.

Bray, E.A.: Molecular responses to water deficit. - Plant Physiol. 103: 1035-1040, 1993.

Campos, F., Zamudio, F., Covarrubias, A.A.: Two different late embryogenesis abundant proteins from Arabidopsis thaliana contain specific domains that inhibit Escherichia coli growth. 
- Biochem. biophys. Res. Commun. 342: 406-413, 2006.

Cuevas-Velazquez, C.L., Reyes, J.L., Covarrubias, A.A.: Group 4 late embryogenesis abundant proteins as a model to study intrinsically disordered proteins in plants. - Plant Signal. Behav. 12: e1343777, 2017.

Cuevas-Velazquez, C.L., Saab-Rincón, G., Reyes, J.L., Covarrubias, A.A.: The unstructured $\mathrm{N}$-terminal region of Arabidopsis Group 4 late embryogenesis abundant (LEA) proteins is required for folding and for chaperone-like activity under water deficit. - J. biol. Chem. 291: 10893-10903, 2016.

Dang, N.X., Popova, A.V., Hundertmark, M., Hincha, D.K.: Functional characterization of selected LEA proteins from Arabidopsis thaliana in yeast and in vitro. - Planta 240: 325336, 2014.

Dure, L.: A repeating 11-mer amino acid motif and plant desiccation. - Plant J. 3: 363-369, 1993.

Dure, L., Crouch, M., Harada, J., Ho, T.H., Mundy, J., Quatrano, R., Thomas, T., Sung, Z.R.: Common amino acid sequence domains among the LEA proteins of higher plants. - Plant mol. Biol. 12: 475-486, 1989.

Dure, L., Greenway, S.C., Galau, G.A.: Developmental biochemistry of cotton seed embryogenesis and germination: changing messenger ribonucleic acid populations as shown by in vitro and in vivo protein synthesis. - Biochemistry 20: 4162-4168, 1981.

French-Pacheco, L., Cuevas-Velazquez, C.L., RivillasAcevedo, L., Covarrubias, A.A., Amero, C.: Metal-binding polymorphism in late embryogenesis abundant protein AtLEA4-5, an intrinsically disordered protein. - Peer J. 6: e4930, 2018.

Hu T.Z., Zhou N., Fu M.L., Qin J., Huang X.Y.: Characterization of OsLEA1a and its inhibitory effect on the resistance of $E$. coli to diverse abiotic stresses. - Int. J. Biol. Macromol. 91: 1010-1017, 2016.

Hundertmark, M., Dimova, R., Lengefeld, J., Seckler, R., Hincha, D.K.: The intrinsically disordered late embryogenesis abundant protein LEA18 from Arabidopsis thaliana modulates membrane stability through binding and folding. -Biochim. biophys. Acta 1808: 446-453, 2011.

Hundertmark, M., Hincha, D.K.: LEA (late embryogenesis abundant) proteins and their encoding genes in Arabidopsis thaliana. - BMC Genomics 9: 118, 2008.

Imai, R., Chang, L., Ohta, A., Bray, E.A., Takagi, M.: A leaclass gene of tomato confers salt and freezing tolerance when expressed in Saccharomyces cerevisiae. - Gene 170: 243-248, 1996.

Kikawada, T., Nakahara, Y., Kanamori, Y., Iwata, K., Watanabe, M., McGee, B., Tunnacliffe, A., Okuda, T.: Dehydrationinduced expression of LEA proteins in an anhydrobiotic chironomid. - Biochem. biophys. Res. Comun. 348: 56-61, 2006.

Liu, G., Xu, H., Zhang, L., Zheng, Y.: Fe binding properties of two soybean (Glycine $\max$ L.) LEA4 proteins associated with antioxidant activity. - Plant Cell Physiol. 52: 994-1002, 2011.

Liu, Y., Wang, L., Xing, X., Sun, L.P, Pan, J.W, Kong, X.P., Zhang, M.Y, Li, D.Q.: ZmLEA3, a multifunctional group 3 LEA protein from maize (Zea mays L.), is involved in biotic and abiotic stresses. - Plant Cell Physiol. 54: 944-959, 2013.
Mota, A.P.Z., Oliveira, T.N., Vinson, C.C., Williams, T.C.R., Costa, M.M.dC., Araujo, A.C.G., Danchin, E.G.J., Grossi-deSá, M.F., Guimaraes, P.M., Brasileiro, A.C.M.: Contrasting effects of wild Arachis dehydrin under abiotic and biotic stresses. - Front. Plant Sci. 10: 497, 2019.

Olvera-Carrillo, Y., Campos, F., Reyes, J.L., Garciarrubio, A., Covarrubias, A.A.: Functional analysis of the Group 4 late embryogenesis abundant proteins reveals their relevance in the adaptive response during water deficit in Arabidopsis. Plant Physiol. 154: 373-390, 2010.

Receveur-Bréchot, V., Bourhis, J.M., Uversky, V.N., Canard, B., Longhi, S.: Assessing protein disorder and induced folding. Proteins (Structure, Function, and Bioinformatics) 62: 24-45, 2006.

Reyes, J.L., Campos, F., Wei, H., Arora, R., Yang, Y., Karlson, D.T., Covarrubias, A.A.: Functional dissection of hydrophilins during in vitro freeze protection. - Plant Cell Environ. 31: 1781-1790, 2008

Reyes, J.L., Rodrigo, M.J., Colmenero-Flores, J.M., Gil, J.V., Garay-Arroyo, A., Campos, F., Salamini, F., Bartels, D., Covarrubias, A.A.: Hydrophilins from distant organisms can protect enzymatic activities from water limitation effects in vitro. - Plant Cell Environ. 28: 709-718, 2005.

Rivera-Najera, L.Y., Saab-Rincón, G., Battaglia, M., Amero, C., Pulido, N.O., García-Hernández, E., Solórzano, R.M., Reyes, J.L., Covarrubias, AA.: A Group 6 late embryogenesis abundant protein from common bean is a disordered protein with extended helical structure and oligomer-forming properties. - J. biol. Chem. 289: 31995-32009, 2014.

Salleh, F.M., Evans, K., Goodall, B., Machin, H., Mowla, S.B., Mur, L.A., Runions J., Theodoulou F.L., Foyer C.H., Rogers, H.J.: A novel function for a redox-related LEA protein (SAG21/AtLEA5) in root development and biotic stress responses. - Plant Cell Environ. 35: 418-429, 2012.

Saucedo A.L., Hernández-Domínguez E.E., De Luna-Valdez L.A., Guevara-García A.A., Escobedo-Moratilla A., Bojorquéz-Velázquez E., Del Río-Portilla F., FernándezVelasco D.A., Barba de la Rosa A.P.: Insights on structure and function of a late embryogenesis abundant protein from Amaranthus cruentus: an intrinsically disordered protein involved in protection against desiccation, oxidant conditions, and osmotic Stress. - Front. Plant Sci. 8: 497, 2017.

Wise, M.J.: LEAping to conclusions: a computational reanalysis of late embryogenesis abundant proteins and their possible roles. - BMC Bioinformatics 4: 52, 2003.

Yang, Y.Z., He, M.Y., Zhu, Z.G., Li, S.X., Xu, Y., Zhang, C.H., Singer, S.D., Wang, Y.J.: Identification of the dehydrin gene family from grapevine species and analysis of their responsiveness to various forms of abiotic and biotic stress. BMC Plant Biol. 12: 140, 2012.

Zhang, L., Ohta, A., Takagi, M., Imai, R.: Expression of plant group 2 and group 3 lea genes in Saccharomyces cerevisiae revealed functional divergence among LEA proteins. - J. Biochem. 127: 611-616, 2000.

Zhang, L.L., Zhao, M.G., Tian, Q.Y., Zhang, W.H.: Comparative studies on tolerance of Medicago truncatula and Medicago falcata to freezing. - Planta 234: 445-457, 2011. 